\title{
Excitonic effects in third-harmonic generation: The case of carbon nanotubes and nanoribbons
}

\author{
C. Attaccalite, ${ }^{1,2,3}$ E. Cannuccia, ${ }^{3,4}$ and M. Grüning ${ }^{3,5}$ \\ ${ }^{1}$ CNRS/Aix-Marseille Université, Centre Interdisciplinaire de Nanoscience de Marseille, UMR 7325, Campus de Luminy, \\ 13288 Marseille Cedex 9, France \\ ${ }^{2}$ CNRS/Université Grenoble Alpes, Institut Néel, F-38042 Grenoble, France \\ ${ }^{3}$ European Theoretical Spectroscopy Facilities \\ ${ }^{4}$ PIIM, Aix-Marseille Université, Marseille, France \\ ${ }^{5}$ School of Mathematics and Physics, Queen's University Belfast, Belfast BT7 1NN, United Kingdom
}

(Received 25 July 2016; revised manuscript received 8 February 2017; published 2 March 2017)

\begin{abstract}
Linear and nonlinear optical properties of low-dimensional nanostructures have attracted great interest from the scientific community as tools to probe the strong confinement of electrons and for possible applications in optoelectronic devices. In particular it has been shown that the linear optical response of carbon nanotubes [F. Wang et al., Science 308, 838 (2005)] and graphene nanoribbons [Nat. Commun. 54253 (2014)] is dominated by bounded electron-hole pairs, excitons. The role of excitons in linear response has been widely studied, but still, little is known about their effect on nonlinear susceptibilities. Using a recently developed methodology [Phys. Rev. B 88, 235113 (2013)] based on well-established ab initio many-body perturbation theory approaches, we find that quasiparticle shifts and excitonic effects significantly modify the third-harmonic generation in carbon nanotubes and graphene nanoribbons. For both systems the net effect of many-body effects is to reduce the intensity of the main peak in the independent-particle spectrum and redistribute the spectral weight among several excitonic resonances.
\end{abstract}

DOI: 10.1103/PhysRevB.95.125403

\section{INTRODUCTION}

Carbon nanotubes (CNTs) and graphene nanoribbons (GNRs) have remarkable electronic and optical properties due to their one-dimensional structure that combines solidstate characteristics with molecular dimensions. In these nanostructures light absorption produces strongly correlated electron-hole states in the form of excitons. Evidence of excitons has emerged from experimental studies of optical spectra and excited-state dynamics [1,2]. The key role of excitons in the interpretation of the optical absorption of these materials has been confirmed by ab initio computational studies [3,4] based on many-body perturbation theory (MBPT) $[5,6]$. In fact, the formation of strongly bounded excitons in GNRs was theoretically predicted [3] before experimental measurement [2]. In recent years, also the nonlinear optical response of these low-dimensional structures has attracted great attention from both fundamental and applicative points of view. In particular due to their strong nonlinear response, onedimensional nanostructures have application as nanoantennas and optical switches $[7,8]$.

Experimentally, the absolute measure of nonlinear optical responses of these nanostructures is, however, not straightforward [9]. The first nonzero nonlinear response function in carbon nanoribbon and nonchiral nanotubes is the third-order susceptibility.

In CNTs, only a few measurements on third-order nonlinear susceptibility have been reported so far, with most of them on $\chi^{(3)}(-\omega ; \omega,-\omega, \omega)$ [10-13], which is responsible for the intensity dependence of the refractive index. The only study on the third-harmonic generation $\chi^{(3)}(-3 \omega ; \omega, \omega, \omega)$ $[14,15]$ explored the nonperturbative regime. Regarding the GNRs, to our knowledge there are no available experimental measures of the third-harmonic generation (THG), although, recently, measurements of the THG in graphene have been obtained [16-18]. Interestingly, these measurements found that graphene's THG is of the order of $10^{-15}-10^{-16} \mathrm{~m}^{2} / \mathrm{V}^{2}$ $\left(10^{-7}-10^{-8} \mathrm{esu}\right)$ and thus comparable to resonant THG in bulk materials.

Theoretically, state-of-the-art calculations of nonlinear optical responses of periodic systems neglect excitonic effects that are deemed essential to describe optical properties in these carbon nanostructures. For CNTs theoretical studies on THG, which mostly focused on radius and chirality dependence, have been performed with the independent-particle approximation [19-21]. The only work which includes many-body effects to our knowledge is from Lacivita and coworkers [22], who computed the static second hyperpolarizability of CNTs within the coupled Kohn-Sham (KS) equation formalism [23]. For GNRs the nonlinear properties have been addressed in a few studies [24-27] that focused mainly on the possibility of enhancing the nonlinear response by engineering the ribbon edges [25,26]. The works in Refs. [25,26] are based on time-dependent density-functional theory with a hybrid approximation for the exchange-correlation functional, which approximately accounts for excitonic effects. Those studies, however, have been performed on finite-length GNRs.

Here we present a theoretical/computational combined study of THG in CNTs and GNRs (Fig. 1) that includes excitonic effects. Specifically, we use an $a b$ initio approach based on MBPT, which has been shown to provide accurate results for both linear and nonlinear optical properties of periodic systems $[5,6,28,29]$.

With respect to the works mentioned above which include correlation beyond the independent-particle approximation, our study addresses infinite periodic nanostructures (differently from Refs. [25,26]) and frequency-dependent thirdorder response (differently from Ref. [22]). Furthermore our MBPT approach does not depend on any semiempirical 


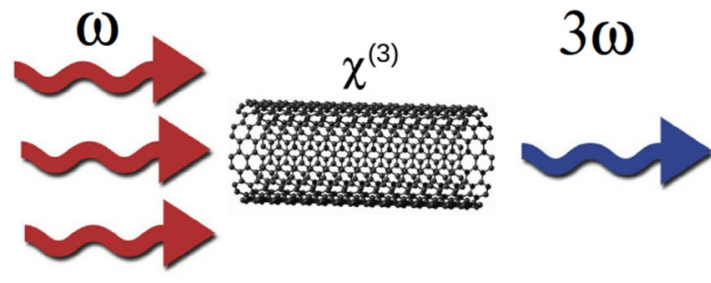

FIG. 1. Schematic description of the THG process: three photons of frequency $\omega$ are destroyed, and one photon of frequency $3 \omega$ is created; that is, the system responds at frequency $3 \omega$ to an applied field at frequency $\omega$.

parameter, which is different from hybrid density-functionalbased approaches used in previous works. In the latter, the semiempirical mixing parameter eventually determines both the fundamental band gap and the amount of dielectric screening.

The purpose of this work is threefold: (i) to provide an accurate theoretical estimate of the THG in a small semiconducting CNT and GNR; (ii) more importantly, as we can switch on and off excitonic and many-body effects in the (effective) Hamiltonian of the electron system, to evaluate how those effects affect the THG of the material; and (iii) to provide a benchmark to assess the reliability and accuracy of calculations at the independent-particle level which neglects these effects. This paper is organized as follows: in Sec. II we summarize the computational methods employed in the calculation of the electronic structure and nonlinear response; in Sec. III we present results for both the CNTs and GNRs. We consider here only centrosymmetric systems for which the THG is the first non-negligible nonlinear response [30].

\section{COMPUTATIONAL METHODS}

The nanotube and nanoribbon atomic structures were generated from ideal graphene with a bonding length of $1.421 \AA$ A. Subsequently, atomic positions were optimized by means of density-functional theory (DFT), using the localdensity approximation for the exchange-correlation functional [31,32].

All DFT calculations have been performed with the QUANTUM ESPRESSO code [33], where the wave functions are expanded in plane waves with a cutoff of $60 \mathrm{Ry}$ and the effect of core electrons is simulated by norm-conserving pseudopotentials [34]. We used a $1 \times 1 \times 22 \mathbf{k}$-point grid to converge the density in the CNT and GNR (both structures are oriented along the $z$ axis). Valence and conduction orbitals that enter in the Green's function theory are obtained from the diagonalization of the KS eigensystem. The KS eigensolutions $\left\{\varepsilon_{n \mathbf{k}} ;|n \mathbf{k}\rangle\right\}$ correspond to the energies and Bloch wave functions (with $\mathbf{k}$ being the crystal wave vector and $n$ being the band index) of the independent-particle system that reproduces the electronic density of the system under study.

In order to simulate isolated nanotubes and nanoribbons we used a supercell approach with a tube-tube distance larger than the nanotube diameter and a distance between ribbons of 16 a.u. in the perpendicular direction and larger than the ribbon size in the plane.

\section{A. Quasiparticle band structure}

Starting from the KS eigenvalues and eigenfunctions, we obtained the quasiparticle (QP) band structure by means of MBPT within the so-called $G W$ approximation. Specifically, we use non-self-consistent $G W$, often denoted as $G_{0} W_{0}$, in which the screened Coulomb potential $W$ and the Green's function $G$ are built from the KS eigensolutions $\left\{\varepsilon_{n \mathbf{k}} ;|n \mathbf{k}\rangle\right\}$ and the quasiparticle energies are obtained from

$$
\varepsilon_{n \mathbf{k}}^{\mathrm{QP}}=\varepsilon_{n \mathbf{k}}+Z_{n \mathbf{k}} \Delta \Sigma_{n \mathbf{k}}\left(\varepsilon_{n \mathbf{k}}\right) .
$$

In Eq. (1)

$$
Z_{n \mathbf{k}}=\left[1-\partial \Delta \Sigma_{n \mathbf{k}}(\omega) /\left.\partial \omega\right|_{\omega=\varepsilon_{n \mathbf{k}}}\right]^{-1}
$$

is the renormalization factor, and

$$
\Delta \Sigma_{n \mathbf{k}} \equiv\langle n \mathbf{k}|\Delta \hat{\Sigma}| n \mathbf{k}\rangle
$$

where

$$
\Delta \hat{\Sigma}=\hat{\Sigma}-\hat{V}^{\mathrm{xc}}
$$

is the difference between $\Sigma=G W$, the $G W$ self-energy, and $V^{\mathrm{xc}}$, the exchange-correlation functional used in the KS calculation [6]. The screened Coulomb potential $W$ has been evaluated within the random-phase approximation. In the $G W$ approach we used the Godby-Needs plasmon-pole model to approximate the dynamical behavior of $W$ [35], while in the Bethe-Salpeter equation (BSE) framework described in the next section we use the static approximation [5]. In the $G W$ (and BSE) calculations we used a truncated Coulomb potential to reduce the interaction between the periodic replica [36]. The Green's function and the self-energy that appear in the $G W$ calculations are expanded in the basis of the KS eigensolutions: we used $40 \mathrm{k}$ points and 320 bands for the CNT and $60 \mathbf{k}$ points and 200 bands for the GNR.

\section{B. Linear and nonlinear response functions}

Linear and nonlinear optical properties are obtained by means of a real-time implementation of the BSE [5,37]. We used an effective Hamiltonian that includes electron-hole interaction through a screened exchange interaction, and the coupling between electrons and the external field is described by means of the modern theory of polarization [38,39]. This formulation allows us to correctly describe response functions beyond the linear one [39].

Specifically, we solve a set of coupled one-particle effective time-dependent Schrödinger equations:

$$
i \hbar \frac{d}{d t}\left|v_{m \mathbf{k}}\right\rangle=\left(H_{\mathbf{k}}^{\mathrm{sys}}+i \mathcal{E} \cdot \tilde{\partial}_{\mathbf{k}}\right)\left|v_{m \mathbf{k}}\right\rangle,
$$

where $\left|v_{m \mathbf{k}}\right\rangle$ is the periodic part of the Bloch states that determine the system polarization [38] as discussed below. In the right-hand side of Eq. (2), $H_{\mathbf{k}}^{\text {sys }}$ is the system Hamiltonian, which is discussed later in this section; the second term, $\mathcal{E} \cdot \tilde{\partial}_{\mathbf{k}}$, describes the coupling with the external field $\mathcal{E}$ in the dipole approximation. As we imposed Born-von Kármán periodic boundary conditions, the coupling takes the form of a k-derivative operator $\tilde{\partial}_{\mathbf{k}}$. The tilde indicates that the operator is "gauge covariant" and guarantees that the solutions of Eq. (2) are invariant under unitary rotations among occupied states at $\mathbf{k}$ (see Ref. [38] for a discussion on this point). 
From $\left|v_{m \mathbf{k}}\right\rangle$, the time-dependent polarization of the system $P_{\|}$along the lattice vector $\mathbf{a}$ is calculated as

$$
P_{\|}=-\frac{e f|\mathbf{a}|}{2 \pi \Omega_{c}} \operatorname{Im} \ln \prod_{\mathbf{k}}^{N_{\mathbf{k}}-1} \operatorname{det} S(\mathbf{k}, \mathbf{k}+\mathbf{q}),
$$

where $S(\mathbf{k}, \mathbf{k}+\mathbf{q})$ is the overlap matrix between the valence states $\left|v_{n \mathbf{k}}\right\rangle$ and $\left|v_{m \mathbf{k}+\mathbf{q}}\right\rangle$. Furthermore, $\Omega_{c}$ is the unit-cell volume, $f$ is the spin degeneracy, $N_{\mathbf{k}}$ is the number of $\mathbf{k}$ points along the polarization direction, and $\mathbf{q}=2 \pi /\left(N_{\mathbf{k}} \mathbf{a}\right)$. Finally, the third-harmonic coefficient is extracted from the expansion of the polarization in the laser field $\mathcal{E}$ power series:

$$
\mathbf{P}=\chi^{(1)} \mathcal{E}+\chi^{(2)} \mathcal{E}^{2}+\chi^{(3)} \mathcal{E}^{3}+\cdots,
$$

as detailed in Ref. [39].

Notice that our approach to calculate nonlinear susceptibilities does not work for metallic systems or systems with a very small gap since it uses the polarization [Eq. (3)] as a fundamental quantity. For metallic systems other approaches based on the electron current density should be used instead [40].

In Eq. (2) the model Hamiltonian chosen for $H_{\mathbf{k}}^{\text {sys }}$ determines the level of approximation in the description of correlation effects in the linear and nonlinear spectra. In this work we use different models for the system Hamiltonian: (i) the independent-particle (IP) model,

$$
H_{\mathbf{k}}^{\mathrm{IP}} \equiv H_{\mathbf{k}}^{\mathrm{KS}},
$$

where $H_{\mathbf{k}}^{\mathrm{KS}}$ is the unperturbed $\mathrm{KS}$ Hamiltonian and we consider the KS system to be a system of independent particles; (ii) the QP model,

$$
H_{\mathbf{k}}^{\mathrm{QP}} \equiv H_{\mathbf{k}}^{\mathrm{KS}}+\Delta H_{\mathbf{k}},
$$

where a scissor operator $\Delta H_{\mathbf{k}}$, estimated from the MBPT [Eq. (1)], has been applied to the KS eigenvalues; and (iii) the full $G W+\mathrm{BSE}$ model,

$$
H_{\mathbf{k}}^{G W+\mathrm{BSE}} \equiv H_{\mathbf{k}}^{\mathrm{KS}}+\Delta H_{\mathbf{k}}+V_{h}(\mathbf{r})[\Delta \rho]+\Sigma_{\mathrm{SEX}}[\Delta \gamma],
$$

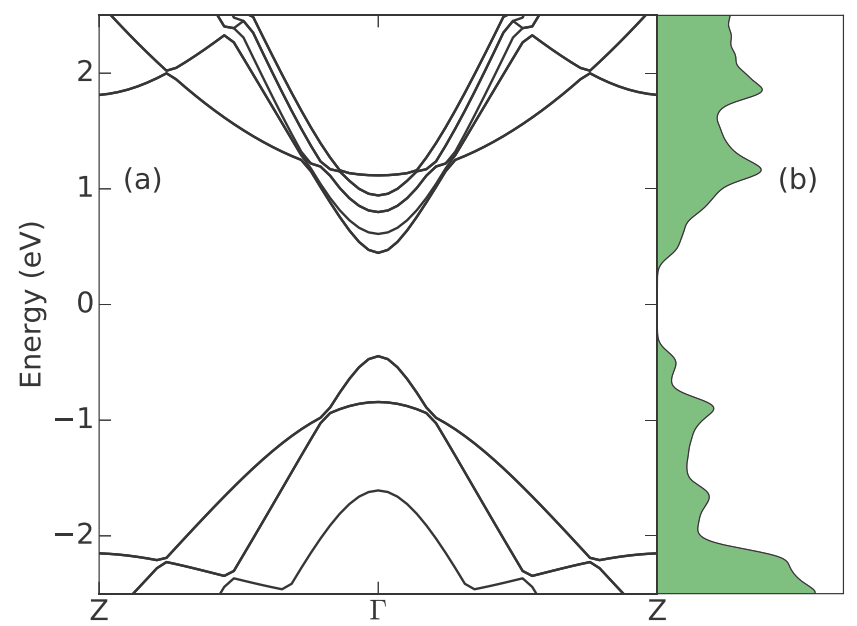

where

$$
\Delta \rho \equiv \rho(\mathbf{r} ; t)-\rho(\mathbf{r} ; t=0)
$$

is the variation of the electronic density and

$$
\Delta \gamma \equiv \gamma\left(\mathbf{r}, \mathbf{r}^{\prime} ; t\right)-\gamma\left(\mathbf{r}, \mathbf{r}^{\prime} ; t=0\right)
$$

is the variation of the density matrix induced by the external field $\mathcal{E}$.

In Eq. (7) the term $V_{h}(\mathbf{r})[\Delta \rho]$ is the time-dependent Hartree potential [41] and is responsible for the local-field effects [42] originating from system inhomogeneities. In the same equation, $\Sigma_{\mathrm{SEX}}$ is the screened-exchange self-energy that accounts for the electron-hole interaction [5] and is given by the convolution between the screened interaction $W$ and $\Delta \gamma$. In the limit of small perturbation Eq. (7) reproduces the optical absorption calculated with the standard $G W+$ BSE approach [5], as shown both analytically and numerically in Ref. [41].

We calculate $\left|\chi_{z z z z}^{(3)}(-3 \omega ; \omega ; \omega ; \omega)\right|$, i.e., the magnitude of the third-order nonlinear susceptibility, at a frequency of $3 \omega$ along the $z$ axis (the orientation axis of nanotubes and the ribbons) when a monochromatic electric field of frequency $\omega$ is applied along $z$. This quantity is obtained by integrating Eq. (2) numerically for a time interval of $120 \mathrm{fs}$ using the numerical approach described in Ref. [38] (originally taken from Ref. [43]) with a time step of $\Delta t=0.01 \mathrm{fs}$, which guarantees numerically stable and sufficiently accurate simulations. $\left|\chi_{z z z z}^{(3)}\right|$ is finally extracted from the total polarization after $100 \mathrm{fs}$, as described in Ref. [39]. A dephasing term with a time $\tau=8.78$ fs is introduced in Eq. (2) in order to simulate a finite broadening of about $0.15 \mathrm{eV}$ [39]. Finally, note that our calculations provide the volume third-order nonlinear susceptibility, but because of the use of the supercell, two of the dimensions are not physical. We then rescale by the effective physical dimensions of the systems: for both systems we use $0.335 \mathrm{~nm}$ as the effective thickness, and for the GNRs we consider as the effective width the width of the ribbon plus $0.2 \mathrm{~nm}$.

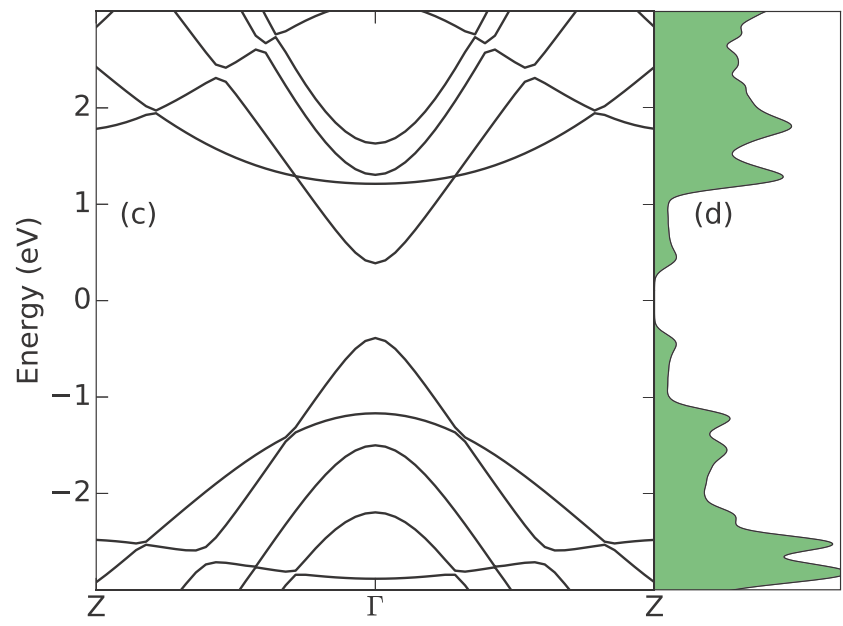

FIG. 2. Band structure and density of states for (a) and (b) the (10,0) CNT and (c) and (d) 9-AGNR. Notice that the band structures and densities of states are aligned in such a way that they have the same $y$ axis. The density of states is normalized in such a way that its integral on the occupied bands give the total number of electrons. 


\section{RESULTS}

\section{A. Carbon nanotube}

A single-walled CNT is formed by rolling a sheet of graphene into a cylinder along an $(m, n)$ lattice vector in the graphene plane. These two indexes determine the diameter and chirality, which are key parameters of a nanotube. Depending on the chirality (the chiral angle between hexagons and the main CNT axis), nanotubes can be either metals or semiconductors, with band gaps that may vary between a few $\mathrm{meV}$ and an $\mathrm{eV}$, even if they have nearly identical diameters [44]. We consider here only semiconducting zigzag CNTs.

We analyze the effect of correlation in THG within the $G W+$ BSE approach, as detailed in Sec. II B. Due to the computational cost we limit the calculation to the $(10,0) \mathrm{CNT}$. We expect the analysis to be valid for larger zigzag CNTs.

In Fig. 3 we report the THG magnitude $\left|\chi_{z z z z}^{(3)}(\omega)\right|$ at different levels of approximation [Figs. 3(a) and 3(c)] and the corresponding optical absorption [Figs. 3(b) and 3(d)] to identify the resonances in the THG spectrum from the comparison.

At the IP level [Fig. 3(b)] the absorption spectrum is dominated by a large peak at about $0.9 \mathrm{eV}$ corresponding to the transition between the highest valence and lowest conduction bands at $\Gamma$ [see band structure, Fig. 2(a)]. Transitions between the second-highest valence and the lowest conduction bands give rise to a shoulder around $1.3 \mathrm{eV}$ that is visible also in the THG spectra. Peaks at higher energies originate from transitions from the third-highest valence bands.

Turning to the THG, at the level of the IP model [Fig. 3(a)] we observe at about 0.3 and $0.4 \mathrm{eV}$ three-photon resonances corresponding to the main peaks in the absorption spectrum. Notice that in the THG the intensity of the peaks is reversed with respect to the linear optics. At $0.6 \mathrm{eV}$ we found a smaller three-photon resonance peak that stems from the transition from the third-highest valence band. One-photon resonance peaks are barely visible on this scale. Our results are consistent with those obtained by Nemilentsau et al. [15] and $\mathrm{Xu}$ and Xiong [45]. With respect to Nemilentsau and coworkers our calculated linear and nonlinear response functions have a richer structure as we use a full ab initio band structure rather than the two-band model employed in their work.

Inclusion of QP corrections rigidly blueshifts the absorption spectrum by approximately $1.6 \mathrm{eV}$ (not shown). When one includes as well the electron-hole interaction $[G W+\mathrm{BSE}$ model in Fig. 3(d)], the spectrum is redshifted with respect to the QP model by $1.5 \mathrm{eV}$ (excitonic binding energy). As a result the resonance peak is blueshifted by about $0.1 \mathrm{eV}$ with respect to the IP level. More remarkably, the spectrum is dominated by a strong excitonic peak at $1.1 \mathrm{eV}$ which almost doubles in intensity the Van Hove singularity in the IP spectrum.

In the THG spectrum the inclusion of QP corrections blueshifts it by about $0.5 \mathrm{eV}$ and reduces the spectral intensity by almost one order of magnitude due to the sum-rule constraints [46]. Similar to what is observed for the absorption spectrum, the inclusion of electron-hole interaction [Fig. 3(c)] redshifts the spectrum and enhances the spectral intensity with respect to the QP model. The spectral enhancement is, however, not uniform: the intensity of the main peak is
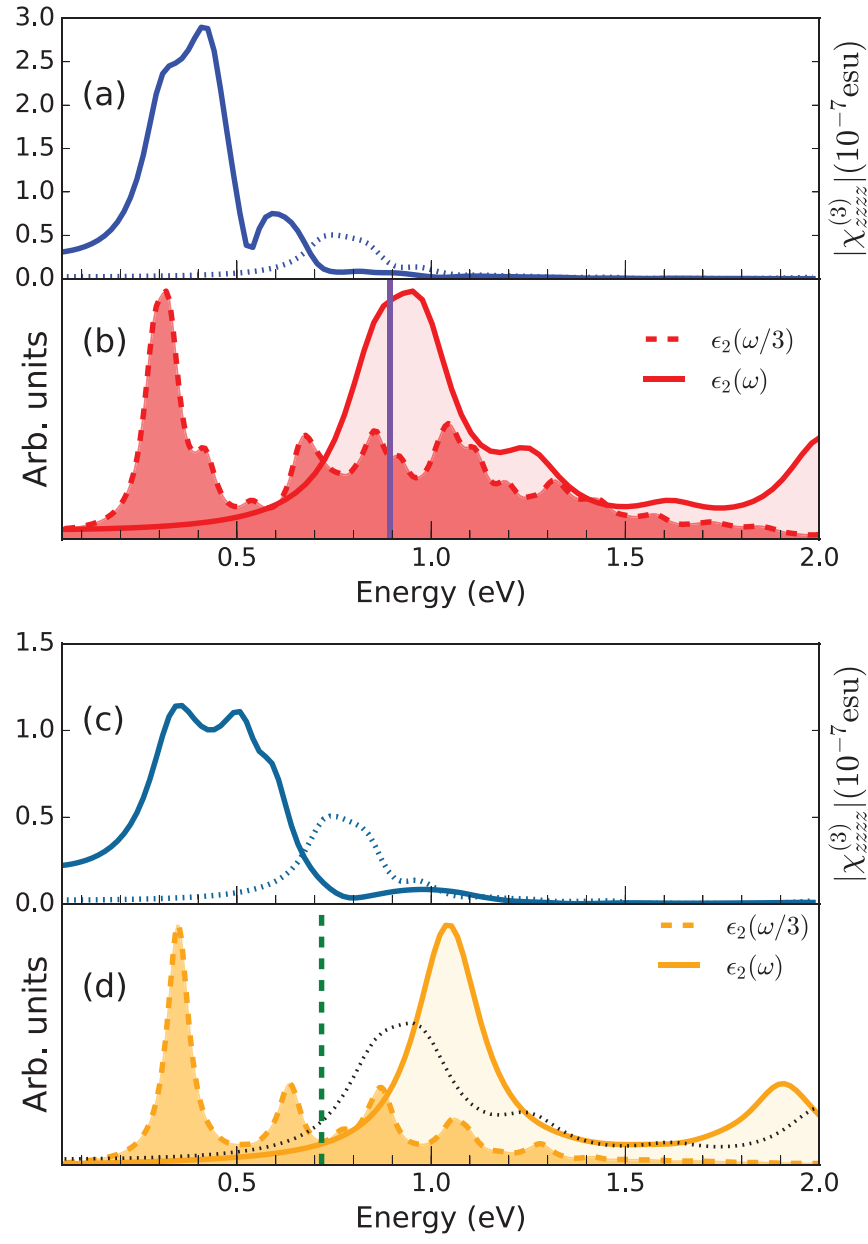

FIG. 3. THG intensity $\left|\chi_{z z z z}^{(3)}(\omega)\right|$ and optical absorption in the longitudinal direction ( $z$ axis) for the $(10,0)$ CNT. (a) THG from the IP model (solid line) and the QP model (dotted line) for the Hamiltonian. (b) The imaginary part of the dielectric function at $\omega$ (solid line) and $\omega / 3$ (dashed line) from the IP model for the Hamiltonian. (c) THG within the GW+BSE model (solid line) and QP model (dotted line) Hamiltonian. (d) The imaginary part of the dielectric function at $\omega$ (solid line) and $\omega / 3$ (dashed line) within the GW+BSE model and the IP results at $\omega$ (black dotted line). The vertical violet line represents the KS fundamental gap, and the dashed line represents $1 / 3$ of the $G W$ fundamental gap.

doubled, although it is still about $1 / 3$ of the main peak intensity in the IP model. On the other hand, peaks at higher frequencies acquire an intensity comparable to the main peak. The onephoton resonance with the main peak remains very weak.

Notice that the $G W+$ BSE calculations include also the so-called local-field effects, namely, the response of the time-dependent Hartree term to the external field. These corrections are large for inhomogeneous systems, such as isolated molecules or localized orbitals, and are exactly zero for a homogeneous electron gas. In the linear response of one-dimensional systems (e.g., CNTs) local-field effects are negligible along the periodic direction and large for the perpendicular directions. We found that in the periodic direction the local-field effects are negligible for the THG as well.

Finally, at only the IP level we study how the THG varies with the size of the CNT [Fig. 4(a)]. Consistent with what was 

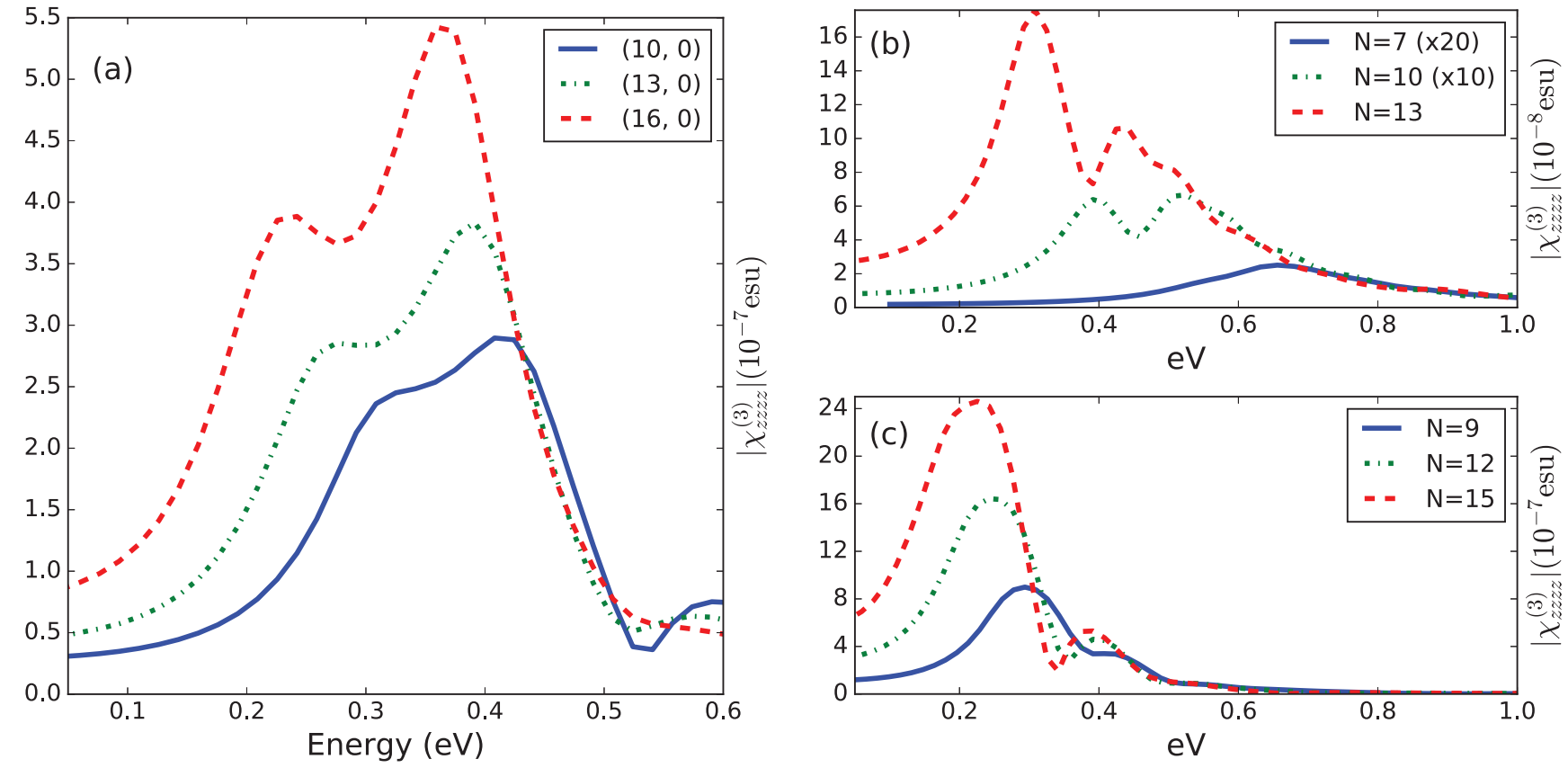

FIG. 4. THG response in GNRs and CNTs at the IP level. (a) THG for zigzag CNTs of increasing size. (b) THG for the $N=3 p+1$ GNR family and (c) THG for the $N=3 p$ family.

found previously $[19,20,45]$, the THG increases superlinearly with the $\mathrm{CNT}$ radius. The resonant peaks shift to lower energy as a consequence of the gap shrinking for larger CNTs. The two peaks, however, do not shift the same amount, and they become farther apart in larger CNTs. We found a factor of about 1.4 between the peaks of the $(13,0)$ and $(16,0)$ CNTs, in agreement with Refs. [19,45], which predicted a factor of about 1.5. The peaks' magnitude is in agreement with that of Margulis and Sizikova [19], while others have reported larger magnitude, especially Xu and Xiong [45], whose reported THG at resonance is larger by almost two orders of magnitude. Regarding the spectral shape, it is clear that the two-band model employed in previous works is not sufficient and at least the second-highest valence band needs to be included.

\section{B. Armchair nanoribbon}

Armchair nanoribbons are divided into three distinct families depending on the ribbon width, namely, $N=3 p, N=$ $3 p+1$, and $N=3 p+2$, with $N$ being the number of dimer lines along the width and $p$ being a positive integer. Within each family, the fundamental band gap decreases with increasing ribbon width [47]. We consider here only the semiconducting $N=3 p, N=3 p+1$ families.

We analyze the many-body effects on the THG [Figs. 5(a) and 5(c)] and on the absorption spectrum [Figs. 5(b) and 5(d)] for the armchair GNR with $N=9$ (9-AGNR). Within the IP level of approximation [Eq. (5)] the optical spectrum shows the characteristic one-dimensional Van Hove singularity at about $0.8 \mathrm{eV}$ [Fig. 5(b)] resulting from transitions at the $\Gamma$ point [see band structure, Fig. 2(c)] and two shoulders at about 1.0 and $1.2 \mathrm{eV}$. When the quasiparticle corrections and the electronhole interaction are turned on [ $G W+$ BSE model in Eq. (7)], the absorption spectrum below $2 \mathrm{eV}$ [Fig. 5(d)] presents a single excitonic peak well below the onset of the continuum [which is shifted $1.2 \mathrm{eV}$ by quasiparticle corrections; see vertical lines in Figs. 5(b) and 5(d)] with an exceedingly large excitonic binding energy for semiconducting materials $(1 \mathrm{eV})$. The main peak is enhanced by about $40 \%$ by the electron-hole interaction [48]. To sum up, the inclusion of the quasiparticle corrections and electron-hole interaction modifies the absorption line shape, narrowing the main peak. These effects are indeed known to be important for qualitative and quantitative predictions of the optical spectra [3].

The effect of correlation on the THG intensity is even more dramatic and not entirely predictable from what is observed for absorption. Figure 5(a) shows the third-harmonic intensity obtained from the IP model. The peaks observed in $\chi_{z z z z}^{(3)}$ correspond to three-photon resonances of the Van Hove singularities observed in the absorption, and in fact, the spectral shape roughly resembles that of absorption [Fig. 5(b)]. When we apply QP correction to the KS band structure [QP model in Eq. (6)], the spectrum [Fig. 5(b)] is shifted to higher energies by about $0.35 \mathrm{eV}$, which is roughly $1 / 3$ of the quasiparticle correction to the fundamental band gap (see also Ref. [48]). Since by construction $\chi^{(3)}$ contains the product of terms with poles at different energies the $G W$ correction effect is not a simple energy shift. The spectral shape is modified too. More strikingly, there is a substantial reduction of the peak intensity (about 75\%), as one can expect from sum rule constraints [46].

When excitonic effects are turned on $[G W+$ BSE model, Fig. 5(c)], the spectra are redshifted with respect to the QP model. As observed for the absorption, the cancellation between the QP corrections and the exciton binding energy is partial, and the main peak in the spectrum is slightly blueshifted $(0.05 \mathrm{eV})$ with respect to the IP model. More importantly, the spectral weight is redistributed among a few excitonic three-photon resonances, and the spectral shape is distinctly different from the one obtained from the IP model. The main peak is, in this case, significantly broadened. 

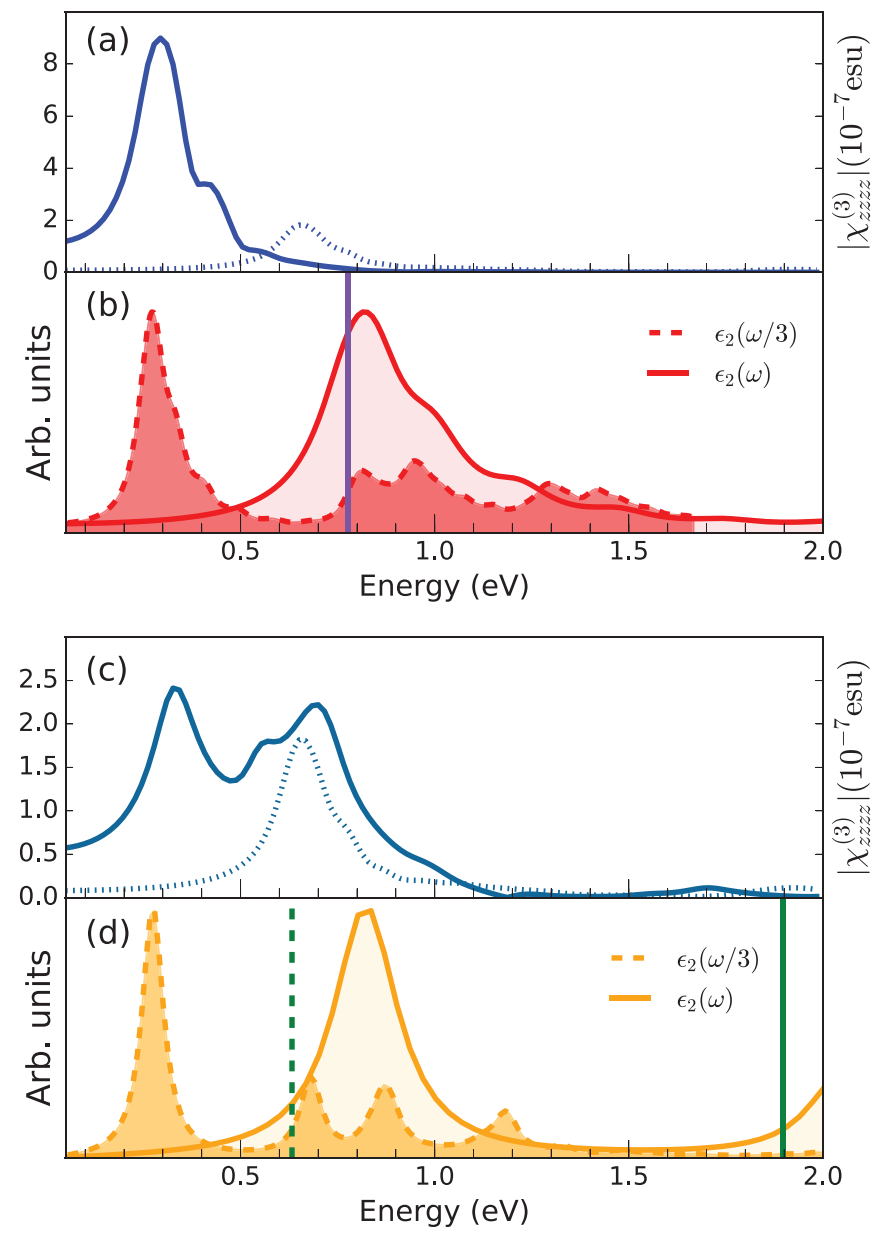

FIG. 5. THG intensity $\left|\chi_{z z z z}^{(3)}(\omega)\right|$ and optical absorption in the longitudinal direction ( $z$ axis) for 9-AGNR. (a) THG from the IP model (solid line) and the QP model (dotted line) for the Hamiltonian. (b) The imaginary part of the dielectric function at $\omega$ (solid line) and $\omega / 3$ (dashed line) from the IP model for the Hamiltonian. (c) THG within the $G W+\mathrm{BSE}$ model (solid line) and QP model (dotted line) Hamiltonian. (d) The imaginary part of the dielectric function at $\omega$ (solid line) and $\omega / 3$ (dashed line) at the same level of approximation. The vertical solid green (violet) line represents the $G W$ (Kohn-Sham) fundamental gap, and the green dashed line represents $1 / 3$ of the $G W$ fundamental gap.

In analogy to the CNTs, we study how the THG varies as a function of the ribbon size (width) for $N=3 p+1$, with $p=2,3,4$ [Fig. 4(b)], and $N=3 p$, with $p=3,4,5$ [Fig. 4(c)]. Similar to what is found for the CNTs, the intensity increases with the size. This is a consequence of the band gap decreasing when the width increases, consistent with the findings in Ref. [27]. We also observe that the THG of the $N=3 p+1$ family, which has the largest band gaps, is smaller by about one to two orders of magnitude than the THG of the $N=3 p$ family, which is related to the suppression of the intraband terms in the THG. Also for $N=3 p+1$ the increase in the THG with the size is more pronounced since the band gap decreases rapidly with $p$. For both families the increase is not uniform but is larger for the first peak. In particular for the $N=3 p$ family the first peak increases by a factor of 2 , passing from $N=9$ to $N=12$, and by a factor 1.5 , passing from $N=12$ to $N=15$.
In both cases it is significantly blueshifted. The shoulder at $0.4 \mathrm{eV}$ in the 9-AGNR instead is only slightly blueshifted and increases in intensity in the larger GNRs.

Finally, our calculations predict a THG of the order of $10^{-7}$ esu at resonances for small GNRs of the same order of magnitude as the THG in graphene [16-18].

\section{DISCUSSION AND CONCLUSIONS}

Using state-of-the-art ab initio MBPT approaches $(G W+$ BSE), we have studied the THG of two paradigmatic onedimensional semiconducting nanostructures, a carbon nanotube and a graphene nanoribbon. By comparing the results from the QP and $G W+$ BSE model Hamiltonians with the simple IP model Hamiltonian we were able to single out the effect of the electron-electron and electron-hole interactions in the THG of these two systems. For both systems the inclusion of many-body effects modifies significantly the THG: first, the intensity of the main peak is reduced by about a factor 3; second, additional structures have a significant spectral weight, and as a result the spectrum has a much richer structure and covers a much larger range of frequencies than its counterpart at the IP level. These results indicate that it is important to include many-body effects for a qualitative and quantitative description of nonlinear optical properties of nanostructures.

Our results agree with what was observed by Lacivita and coworkers in a recent study on static hyperpolarizabilities of carbon nanotubes using a coupled perturbed KS approach [22]. Their calculations show a gradual suppression of the longitudinal hyperpolarizabilities of the carbon nanotube when increasing the nonlocal exchange contribution (Hartree-Fock) to the exchange-correlation potential from $0 \%$ to $100 \%$. In fact, the inclusion of nonlocal exchange in the DFT functional opens the fundamental band gap, similar to the QP corrections in our work, which reduces the third-harmonic intensity. Lacivita and coworkers further observed that the reduction due to the band gap opening is only partially compensated by the inclusion of electron-hole interaction in the response function, in agreement with our findings. Note that in experiments it is difficult, if not impossible, to isolate the effects contributing to the THG. For example, one could, in principle, compare $\chi_{z z z z}^{(3)}$ measured in an isolated CNT with that in CNTs bundles, increasing the dielectric screening and thus "suppressing" the many-body effects. However, other effects, for instance, the variation of the phase relaxation time with the environment, would make the interpretation of these experiments not straightforward [10].

Remarkably, including QP corrections and electron-hole interaction has a very different effect on nanostructure optical absorption spectra: QP corrections usually correspond to a rigid blueshift of the spectra, while electron-hole interaction produces a redshift and a substantial enhancement of the intensity of the first peak, which is usually more intense than the corresponding Van Hove singularity at the IP level. Calculations of the second-harmonic generation $[28,49]$ showed that electron-hole interaction enhances the intensity of the main spectral features by $20 \%$ to $200 \%$ when compared with spectra calculated at the IP level. In the case of secondharmonic generation (SHG), in fact, the addition of $\mathrm{QP}$ corrections shifts the spectrum and reduces the overall spectral 
intensity (as in the case of THG); however, this reduction is overcompensated by the enhancement from the electron-hole interaction.

We argue that the different behaviors of the THG, SHG, and absorption follow from sum-rule constraints [46]. On the other hand, dimensionality could also play an important role since in one-dimensional systems both QP corrections and exciton binding energy are particularly large due to geometrical confinement and poor screening. In this regard it would be of interest to systematically study the THG for systems of different dimensionality. At present this study is hindered by the computational cost of solving the time-dependent Bethe-Salpeter equation [41] for systems with a large number of $\mathbf{k}$ grid points, as is the case of bulk semiconductors. Other approaches, based, for example, on the generalization of time-dependent density-functional theory [50,51] or extension of coupled Kohn-Sham equations to dynamical electric fields [52], could make this kind of study affordable once accuracy issues have been addressed.
Finally, we have also studied the dependence of the THG on the size of the nanostructures (i.e., the radius of the CNT and the width of the GNR). Consistent with previous studies, we have found that THG is increased by increasing the radius and width of the nanostructures, mainly because of the shrinking of the band gap. Our results show as well that the two-band model that has been used in previous studies does not capture the details of the THG spectrum and more bands close to the Fermi energy need to be taken into account.

\section{ACKNOWLEDGMENTS}

Computing time was provided by the national GENGIIDRIS supercomputing centers at Orsay under Contract No. i2012096655. C.A. and M.G. thank the EUspec COST Action for Short Term Scientific Missions that allowed us to initiate and carry out this project. C.A. acknowledges support from the Fondation Aix-Marseille Université, http://fondation.univamu.fr/.
[1] F. Wang, G. Dukovic, L. E. Brus, and T. F. Heinz, The optical resonances in carbon nanotubes arise from excitons, Science 308, 838 (2005).

[2] R. Denk, M. Hohage, P. Zeppenfeld, J. Cai, C. A Pignedoli, H. Söde, R. Fasel, X. Feng, K. Müllen, S. Wang et al., Exciton-dominated optical response of ultra-narrow graphene nanoribbons, Nat. Commun. 5, 4253 (2014).

[3] D. Prezzi, D. Varsano, A. Ruini, A. Marini, and E. Molinari, Optical properties of graphene nanoribbons: The role of manybody effects, Phys. Rev. B 77, 041404 (2008).

[4] C. D. Spataru, S. Ismail-Beigi, L. X. Benedict, and S. G. Louie, Excitonic Effects and Optical Spectra of Single-Walled Carbon Nanotubes, Phys. Rev. Lett. 92, 077402 (2004).

[5] G. Strinati, Riv. Nuovo Cimento 11, 1 (1988).

[6] W. G. Aulbur, L. Jönsson, and J. W Wilkins, Quasiparticle calculations in solids, Solid State Phys. 54, 1 (1999).

[7] Y.-C. Chen, N. R. Raravikar, L. S. Schadler, P. M. Ajayan, Y.-P. Zhao, T.-M. Lu, G.-C. Wang, and X.-C. Zhang, Ultrafast optical switching properties of single-wall carbon nanotube polymer composites at $1.55 \mu \mathrm{m}$, Appl. Phys. Lett. 81, 975 (2002).

[8] S. Tatsuura, M. Furuki, Y. Sato, I. Iwasa, M. Tian, and H. Mitsu, Semiconductor carbon nanotubes as ultrafast switching materials for optical telecommunications, Adv. Mater. 15, 534 (2003).

[9] Y. R. Shen, The Principles of Nonlinear Optics (Wiley Interscience, Hoboken, NJ, 2003).

[10] A. Maeda, S. Matsumoto, H. Kishida, T. Takenobu, Y. Iwasa, M. Shiraishi, M. Ata, and H. Okamoto, Large Optical Nonlinearity of Semiconducting Single-Walled Carbon Nanotubes Under Resonant Excitations, Phys. Rev. Lett. 94, 047404 (2005).

[11] X. Liu, J. Si, B. Chang, G. Xu, Q. Yang, Z. Pan, S. Xie, P. Ye, J. Fan, and M. Wan, Third-order optical nonlinearity of the carbon nanotubes, Appl. Phys. Lett. 74, 164 (1999).

[12] M. Ichida, Y. Kiyohara, S. Saito, Y. Miyata, H. Kataura, and H. Ando, Nonlinear optical properties and phase-relaxation processes in single-walled carbon nanotubes, J. Lumin. 129, 1794 (2009).
[13] J.-S. Lauret, C. Voisin, G. Cassabois, J. Tignon, C. Delalande, $\mathrm{Ph}$. Roussignol, O. Jost, and L. Capes, Third-order optical nonlinearities of carbon nanotubes in the femtosecond regime, Appl. Phys. Lett. 85, 3572 (2004).

[14] C. Stanciu, R. Ehlich, V. Petrov, O. Steinkellner, J. Herrmann, I. V. Hertel, G. Ya. Slepyan, A. A. Khrutchinski, S. A. Maksimenko, F. Rotermund et al., Experimental and theoretical study of third-order harmonic generation in carbon nanotubes, Appl. Phys. Lett. 81, 4064 (2002).

[15] A. M. Nemilentsau, G. Ya. Slepyan, A. A. Khrutchinskii, and S. A. Maksimenko, Third-order optical nonlinearity in singlewall carbon nanotubes, Carbon 44, 2246 (2006).

[16] N. Kumar, J. Kumar, C. Gerstenkorn, R. Wang, H.-Y. Chiu, A. L. Smirl, and H. Zhao, Third harmonic generation in graphene and few-layer graphite films, Phys. Rev. B 87, 121406 (2013).

[17] S.-Y. Hong, J. I. Dadap, N. Petrone, P.-C. Yeh, J. Hone, and R. M. Osgood, Jr., Optical Third-Harmonic Generation in Graphene, Phys. Rev. X 3, 021014 (2013).

[18] A. Säynätjoki, L. Karvonen, J. Riikonen, W. Kim, S. Mehravar, R. A. Norwood, N. Peyghambarian, H. Lipsanen, and K. Kieu, Rapid large-area multiphoton microscopy for characterization of graphene, ACS Nano 7, 8441 (2013).

[19] V. A. Margulis and T. A. Sizikova, Theoretical study of third-order nonlinear optical response of semiconductor carbon nanotubes, Phyica B (Amsterdam, Neth.) 245, 173 (1998).

[20] C.-J. Zhang, K.-X. Guo, and S.-D. Liang, Third harmonic generation of semiconductor carbon nanotubes, Chem. Phys. Lett. 433, 101 (2006).

[21] H. Rezania and N. Daneshfar, Study of third harmonic generation in zigzag carbon nanotubes using the Green's function approach, Appl. Phys. A 109, 503 (2012).

[22] V. Lacivita, M. Rérat, R. Orlando, R. Dovesi, and P. Darco, Longitudinal and transverse hyperpolarizabilities of carbon nanotubes: A computational investigation through the coupledperturbed Hartree-Fock/Kohn-Sham scheme, Theor. Chem. Acc. 135, 81 (2016). 
[23] Note that none of the mentioned studies included temperature effects, except Ref. [21], which includes temperature effects by means of Green's function theory.

[24] Y. Wang and D. R. Andersen, First-principles study of the terahertz third-order nonlinear response of metallic armchair graphene nanoribbons, Phys. Rev. B 93, 235430 (2016).

[25] M. Zhang, G. Li, and L. Li, Graphene nanoribbons generate a strong third-order nonlinear optical response upon intercalating hexagonal boron nitride, J. Mater. Chem. C 2, 1482 (2014).

[26] P. Karamanis and C. Pouchan, Second-hyperpolarizability $(\gamma)$ enhancement in metal-decorated zigzag graphene flakes and ribbons: The size effect, J. Phys. Chem. C 117, 3134 (2013).

[27] E. Ahmadia and A. Asgarib (unpublished), http://graphita.bo. imm.cnr.it/graphita2011/Poster/Ahmadi.pdf.

[28] C. Attaccalite, A. Nguer, E. Cannuccia, and M. Grüning, Strong second harmonic generation in $\mathrm{SiC}, \mathrm{ZnO}, \mathrm{GaN}$ two-dimensional hexagonal crystals from first-principles many-body calculations, Phys. Chem. Chem. Phys. 17, 9533 (2015).

[29] M Grüning and C. Attaccalite, Second harmonic generation in $h-\mathrm{BN}$ and $\mathrm{MoS}_{2}$ monolayers: Role of electron-hole interaction, Phys. Rev. B 89, 081102 (2014).

[30] A centrosymmetric system has an inversion center, and thus the second-harmonic generation is zero within the electric dipole approximation. When multipole corrections are considered, the second-harmonic generation is nonzero but still very small.

[31] J. P. Perdew and A. Zunger, Self-interaction correction to density-functional approximations for many-electron systems, Phys. Rev. B 23, 5048 (1981).

[32] J. P. Perdew and Y. Wang, Accurate and simple analytic representation of the electron-gas correlation energy, Phys. Rev. B 45, 13244 (1992).

[33] P. Giannozzi et al., QUANTUM ESPRESSO: A modular and open-source software project for quantum simulations of materials, J. Phys. Condens. Matter 21, 395502 (2009).

[34] N. Troullier and J. L. Martins, Efficient pseudopotentials for plane-wave calculations, Phys. Rev. B 43, 1993 (1991).

[35] H. N. Rojas, R. W. Godby, and R. J. Needs, Space-Time Method for $A b$ Initio Calculations of Self-Energies and Dielectric Response Functions of Solids, Phys. Rev. Lett. 74, 1827 (1995).

[36] C. A. Rozzi, D. Varsano, A. Marini, E. K. U. Gross, and A. Rubio, Exact Coulomb cutoff technique for supercell calculations, Phys. Rev. B 73, 205119 (2006).

[37] W. G. Schmidt, S. Glutsch, P. H. Hahn, and F. Bechstedt, Efficient $\mathcal{O}\left(N^{2}\right)$ method to solve the Bethe-Salpeter equation, Phys. Rev. B 67, 085307 (2003).

[38] I. Souza, J. Íñiguez, and D. Vanderbilt, Dynamics of Berry-phase polarization in time-dependent electric fields, Phys. Rev. B 69, 085106 (2004).
[39] C. Attaccalite and M. Grüning, Nonlinear optics from an $a b$ initio approach by means of the dynamical Berry phase: Application to second- and third-harmonic generation in semiconductors, Phys. Rev. B 88, 235113 (2013).

[40] M. Springborg and B. Kirtman, Analysis of vector potential approach for calculating linear and nonlinear responses of infinite periodic systems to a finite static external electric field, Phys. Rev. B 77, 045102 (2008).

[41] C. Attaccalite, M. Grüning, and A. Marini, Real-time approach to the optical properties of solids and nanostructures: Timedependent Bethe-Salpeter equation, Phys. Rev. B 84, 245110 (2011).

[42] S. L. Adler, Quantum theory of the dielectric constant in real solids, Phys. Rev. 126, 413 (1962).

[43] Computational Physics: Fortran Version, edited by S. E. Koonin and D. C. Meredith (Perseus Books, United States of America, 2008).

[44] R. E. Smalley, M. S. Dresselhaus, G. Dresselhaus, and P. Avouris, Carbon Nanotubes: Synthesis, Structure, Properties, and Applications, Topics in Applied Physics (Springer, Berlin, 2003), Vol. 80.

[45] Y. Xu and G. Xiong, Third-order optical nonlinearity of semiconductor carbon nanotubes for third harmonic generation, Chem. Phys. Lett. 388, 330 (2004).

[46] J. J. Saarinen, Sum rules for arbitrary-order harmonic generation susceptibilities, Eur. Phys. J. B 30, 551 (2002).

[47] Y.-W. Son, M. L. Cohen, and S. G. Louie, Energy Gaps in Graphene Nanoribbons, Phys. Rev. Lett. 97, 216803 (2006).

[48] D. Prezzi, D. Varsano, A. Ruini, and E. Molinari, Quantum dot states and optical excitations of edge-modulated graphene nanoribbons, Phys. Rev. B 84, 041401 (2011).

[49] T. G. Pedersen and H. D. Cornean, Optical second harmonic generation from Wannier excitons, Europhys. Lett. 78, 27005 (2007).

[50] M. Grüning, D. Sangalli, and C. Attaccalite, Dielectrics in a time-dependent electric field: A real-time approach based on density-polarization functional theory, Phys. Rev. B 94, 035149 (2016).

[51] E. Luppi, H. Hübener, and V. Véniard, $A b$ initio secondorder nonlinear optics in solids: Second-harmonic generation spectroscopy from time-dependent density-functional theory, Phys. Rev. B 82, 235201 (2010).

[52] B. Kirtman, M. Springborg, M. Rrat, M. Ferrero, V. Lacivita, R. Orlando, and R. Dovesi, The linear and nonlinear response of infinite periodic systems to static and/or dynamic electric fields. Implementation in CRYSTAL code, in Proceedings of the International Conference of Computational Methods in Sciences and Engineering 2010 (ICCMSE-2010), AIP Conf. Proc. No. 1642 (AIP, New York, 2015), p. 193 (2016). 